\title{
Impact of Clustering Oral Symptoms in the Pathogenesis of Radiation Caries: A Systematic Review
}

\author{
Adriele Ferreira Gouvêa Vasconcellos ${ }^{a} \quad$ Natália Rangel Palmier ${ }^{a}$ \\ Ana Carolina Prado Ribeiro ${ }^{a, b}$ Ana Gabriela Costa Normando ${ }^{a}$ \\ Karina Morais-Fariab Wagner Gomes-Silvab, c Aljomar José Vechiato Filho ${ }^{b}$ \\ Mario Fernando de Goes ${ }^{a}$ Adriana Franco Paes Leme ${ }^{d}$ Thaís Bianca Brandão ${ }^{b}$ \\ Marcio Ajudarte Lopes ${ }^{\mathrm{a}}$ Philip D. Marsh ${ }^{\mathrm{e}}$ Alan Roger Santos-Silva ${ }^{\mathrm{a}}$ \\ a Oral Diagnosis Department, Piracicaba Dental School, University of Campinas (UNICAMP), Piracicaba, Brazil; \\ ${ }^{\text {b}}$ Department of Dentistry, São Paulo Cancer Institute (ICESP), São Paulo, Brazil; \\ 'Medical School, Nove de Julho University, São Paulo, Brazil; \\ dBrazilian Biosciences National Laboratory, LNBio, CNPEM, Campinas, Brazil; \\ e Department of Oral Microbiology, School of Dentistry, University of Leeds, Leeds, UK
}

\section{Keywords}

Caries · Cancer · Radiotherapy · Chemotherapy $\cdot$ Xerostomia

\begin{abstract}
Radiation-related caries (RRC) is a disease with a high potential for destruction of the dentition, which impairs quality of life in head-and-neck (HN) cancer (HNC) patients who undergo radiotherapy. In light of the recently described "clustering of oral symptoms theory," the present systematic review (PROSPERO CRD42019132709) aims to assess HN and gastrointestinal (Gl) symptom clusters among HNC patients and discusses how these indirect effects of cancer therapy play a pivotal role in the pathophysiology of RRC. The search was performed at PubMed, Scopus, and Embase and resulted in 11 studies that met the inclusion criteria. Data extraction was performed with respect to the presence of $\mathrm{HN} / \mathrm{Gl}$ symptom clusters among $\mathrm{HNC}$ patients. The methodological data of the studies included were assessed using the
\end{abstract}

karger@karger.com

(c) 2020 S. Karger AG, Basel

www.karger.com/cre

Karger
MAStARI and GRADE instruments. The most prevalent reported HN symptoms were dysphagia, xerostomia, and pain. Taste alterations and fatigue were also commonly reported by the patients. Loss of appetite and weight loss were regularly reported in the studies, as well as nausea and vomiting. The results of the present study suggest that HNC treatment generates clusters of oral symptoms, leading to dietary changes, impaired oral hygiene, enamel fragility, and a highly cariogenic oral environment, which may impact the risk for RRC. A better understanding of oral symptom clustering could be of considerable clinical significance for the oral health and quality of life of HNC patients. Therefore, contemporary protocols of RRC prevention must take this broader treatment scenario of symptom clusters such as oral side effects into account.

(c) 2020 S. Karger AG, Basel

A.F.G.V., N.R.P., P.D.M., and A.R.S.-S. contributed equally to this work. 


\section{Introduction}

Radiation-related caries (RRC) is a chronic side effect of head-and-neck (HN) radiotherapy (HNRT) and has a high potential for tooth destruction. Its causes are still not fully understood, and the ability of HNRT to cause direct radiogenic damage to the dentition leading to $\mathrm{RRC}$ is a major topic of discussion in oral oncology [Faria et al., 2014; Lieshout and Bots, 2014].

Recent publications have linked the elevated risk of the clinically aggressive RRC in $\mathrm{HN}$ cancer (HNC) patients to the indirect effects of cancer therapies [Santos-Silva et al., 2009; Sroussi et al., 2017], which were reinforced by increasing evidence that "symptom clusters" may have a pivotal role in several HN chemoradiotherapy (CRT) toxicities [Xiao et al., 2013, 2014]. The so-called "clustering of oral symptoms" has been previously described and is composed of concurrent mucositis, taste changes, oral infections, oral pain, trismus, hyposalivation, altered saliva composition, and shifts in the composition of the oral microbiota, which lead to significant dietary changes, deficient oral hygiene, and the development of a highly cariogenic oral environment, working in synergy to increase the risk for RRC development and progression [SantosSilva, et al., 2009; Ribeiro et al., 2013; Xiao et al., 2013, 2014; Gomes-Silva et al., 2017; Madrid et al., 2017].

Therefore, the aim of this article is to present a systematic review of the recently described "clustering of oral symptoms" [Xiao et al., 2013, 2014] associated with HNC treatment toxicities in an attempt to emphasize that RRC pathophysiology may be part of a broader, multifactorial setting than has been suggested previously.

\section{Materials and Methods}

\section{Study Design}

The present systematic review was conducted following the Guidelines of Preferred Reporting Items for Systematic Reviews and Meta-Analysis (PRISMA) (online suppl. Table 1; for all online suppl. material, see www.karger.com/doi/10.1159/000504878) [Moher et al., 2009] and was registered at the PROSPERO platform CRD42019132709 [Palmier et al., 2019]. The research question was: Is there a specific clustering of oral symptoms associated with HNC treatment that could impact the pathogenesis of radiation caries?

Studies that assessed the presence of treatment-related symptom clusters among HNC patients were selected. The inclusion criteria followed the PICOS strategy: patients - HNC patients; intervention - HNRT or CRT; comparison - HN and gastrointestinal toxicities (GI)-specific toxicities; outcomes - presence and cluster of symptoms from HNC treatment; study design - clinical trials, descriptive, and observational studies.
Studies were excluded for one of the following reasons: (1) no HNC symptoms; (2) psychological/psychiatric disorders; (3) respiratory system disorders; (4) cardiovascular disorders; and (5) other reasons such as studies assessing molecular features of toxicities and studies assessing symptoms of other disorders such as fibromyalgia, for example.

Electronic and systematic searches of scientific studies that assessed the presence of symptoms and symptom clusters from HNC treatment were conducted in April 2019 (last update June 2019). English language restriction was applied, and there was no restriction regarding the year of publication. The databases of Medline/PubMed (https://www.ncbi.nlm.nih.gov/pubmed), Embase (https://www.embase.com/login), and Scopus (https:// www.scopus.com) were screened. Related MeSH (Medical Subject Headings) as well as free terms were combined on different search strategies to find articles. The process was repeated in each database to ensure that any relevant result would not be missed during the identification phase. Two combinations were performed at each database. Complete search strategies are presented in online supplementary Table 2. Additional searches were conducted by reading reference lists from all selected studies to detect other potentially eligible reports that could meet the inclusion criteria.

\section{Study Selection and Data Collection}

All titles were systematically organized using Microsoft Office Excel 2016 (Microsoft Corporation, Redmond, Washington, DC, USA). They were verified and counted to exclude duplicated items. The articles were selected in 2 phases. In phase 1, 2 authors independently reviewed the titles and abstracts, and selected those that apparently met the inclusion criteria. In phase 2, the same authors read the full texts of the selected articles at phase 1 and excluded those that did not meet the inclusion criteria (online suppl. Table 3 ). Any disagreements in the first or second phases were resolved by discussion and mutual agreement between both authors. Studies were classified into the following categories: duplicated, excluded by title, excluded by abstract, excluded by methodology, and included studies. In the end, reports assessed for eligibility were downloaded from databases in full text version, and they were read in detail as PDF files. Studies that omitted relevant methodological information were also excluded from the current review.

The process for methodological data collection involved 2 investigators (A.F.G.V. and N.R.P.). Data were independently extracted by each investigator and then compared; any disagreements were solved by discussion between both investigators. Methodological data extracted from selected studies were related to first author name, year, country, and journal of publication, type of study, number of patients, tumor topography, stage of disease, cancer treatment, mean radiation dose, type of radiotherapy (RT), chemotherapy medications, chemotherapy cycles, treatment-related toxicities, time of assessment, HN-specific symptoms, GI and general symptoms, toxicity assessment criteria, and criteria for inclusion of toxicities in the Results section. The presence of the reported symptoms per included study was assessed.

\section{Risk of Bias within Studies}

Methodologically, the authors evaluated all included studies according to a checklist of the Meta-Analysis of Statistics Assessment and Review Instrument (MAStARI) [The Joanna Briggs Institute, 2014]. The reviewers (A.F.G.V. and N.R.P.) independently 


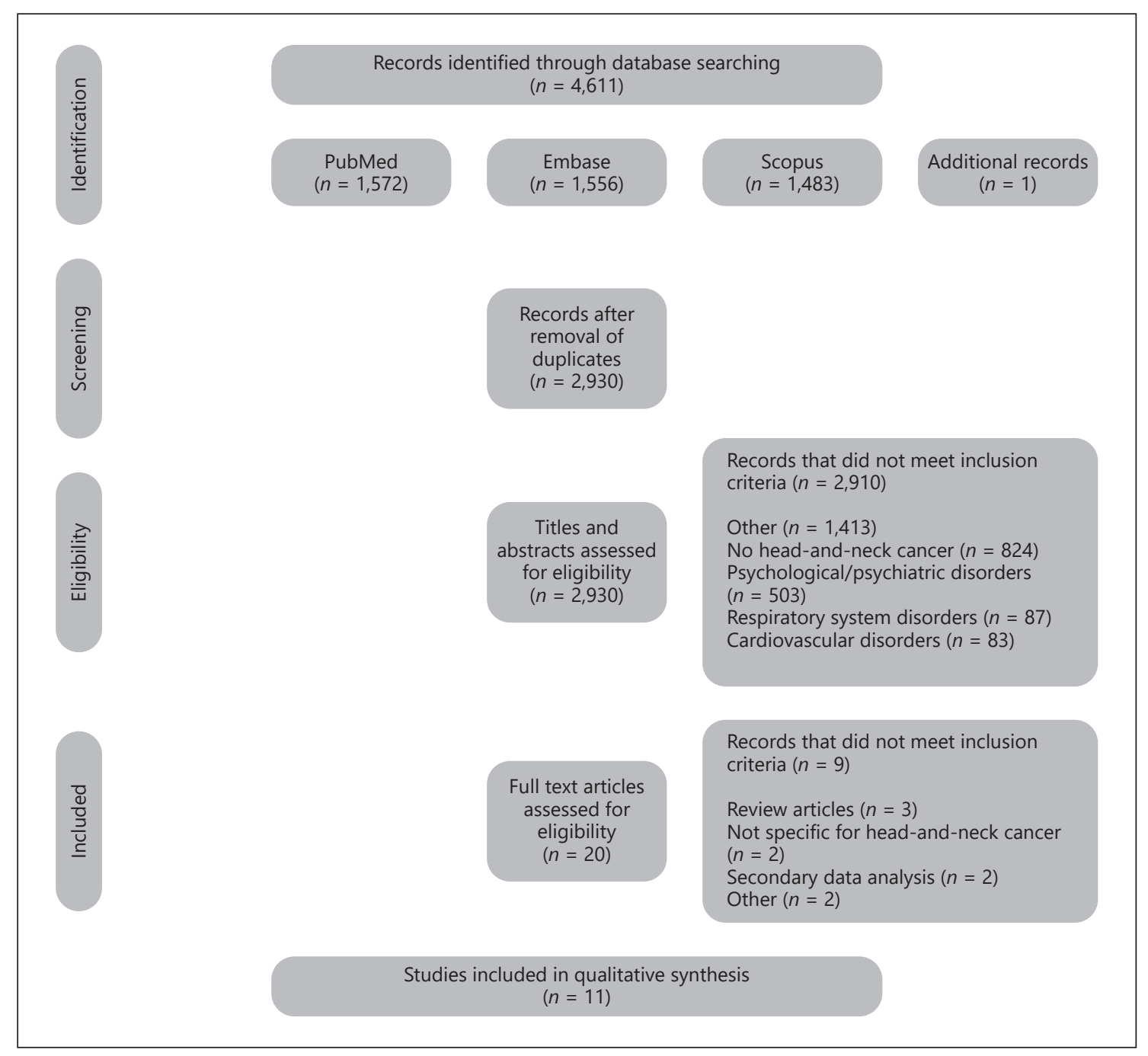

Fig. 1. Flow diagram summarizing the selection process (PRISMA format).

answered 9 questions for descriptive studies and 8 questions for cross-sectional studies as Y for "yes," $\mathrm{N}$ for "no," U for "unclear," and NA for "not applicable" (online suppl. Table 4).

After that, the risk of bias was categorized as high when the study reached up to $49 \%$ of a "yes" score, moderate when the study reached $50-69 \%$ of a "yes" score, and low when the study reached more than $70 \%$ of a "yes" score. Disagreements were resolved by discussion between both authors.

\section{Risk of Bias across Studies}

Quality of evidence and grading of recommendations was assessed by the Grading of Recommendation, Assessment, Development, and Evaluation (GRADE) instrument. The assessment was based on radiation-related symptom clusters evaluated by different study designs. The criteria included the number of studies, study design, risk of bias, inconsistency, indirectness, imprecision, and other considerations such as publication bias and confound- ing factors. Impact, certainty, and importance were graded based on the assessed criteria, and the quality of evidence was characterized as high, moderate, low, or very low for each outcome. The GRADE was assessed using tools from the following website http:// gradepro.org.

\section{Data Analysis}

The primary outcome was to assess the presence of $\mathrm{HN}$-specific symptom clusters. The secondary outcome was to assess the presence of GI symptom clusters. The tertiary outcome was to assess the possible impact of symptom clusters in the pathogenesis of RRC. There was homogeneity in the research purpose among the studies but a great variability in the time of toxicity assessment and criteria used for the assessment of treatment-related toxicities. A detailed qualitative synthesis of the results was performed considering the presence of patient-reported symptoms among the studies included. 


\section{Results}

\section{Study Selection and Characteristics}

A flow diagram summarizing the selection process is shown in Figure 1. A total of 4,611 studies were identified through the search strategies on 3 databases (PubMed, Embase, and Scopus). After the first review process, 1,682 studies were excluded due to interdatabase duplication. One study was added from the search on the reference list of the studies included. A total of 2,919 studies were excluded because they did not meet the inclusion criteria, resulting in 11 studies being eligible for the review. Table 1 shows the main methodological aspects from the 11 studies.

Seven studies (63.6\%) assessed patients with heterogeneous HN topographies [Murphy et al., 2010; Xiao et al., 2013; Rosenthal et al., 2014; Kirca and Kutlutürkan, 2017; Barnhart et al., 2018; Chiang et al., 2018; Ridner et al., 2018], 2 studies (18.2\%) assessed patients with oropharyngeal/laryngeal tumors [Haisfield-Wolfe et al., 2012; Eraj et al., 2017], and 2 studies (18.2\%) assessed patients with nasopharyngeal tumors [Xiao et al., 2017; McDowell et al., 2018]. Eight studies (72.2\%) reported specific information on patients' stage of disease, from which 6 (54.5\%) assessed patients in clinical stage I-IV [Haisfield-Wolfe et al., 2012; Rosenthal et al., 2014; Eraj et al., 2017; Kirca and Kutlutürkan, 2017; Xiao et al., 2017; McDowell et al., 2018] and 2 $(18.2 \%)$ assessed patients with advanced disease (clinical stage III/IV) [Xiao et al., 2013; Chiang et al., 2018].

Information on treatment modalities were also retrieved from the included studies: 7 studies (63.6\%) assessed patients treated with either RT or CRT protocols [Haisfield-Wolfe et al., 2012; Rosenthal et al., 2014; Eraj et al., 2017; Xiao et al., 2017; Barnhart et al., 2018; McDowell et al., 2018; Ridner et al., 2018], 2 studies (18.2\%) assessed patients submitted to RT [Kirca and Kutlutürkan, 2017; Chiang et al., 2018], and 2 studies (18.2\%) assessed patients submitted to CRT protocols [Murphy et al., 2010; Xiao et al., 2013]. Four studies (36.3\%) reported the use of the intensity-modulated RT technique for radiation delivery [Rosenthal et al., 2014; Eraj et al., 2017; Xiao et al., 2017; McDowell et al., 2018], 1 study (7.1\%) reported the use of intensity-modulated RT and 3D conformational RT [Barnhart et al., 2018], and 1 study (7.1\%) compared the outcomes of the accelerated and standard fractionation RT [Xiao et al., 2013]. For the studies that assessed CRT protocols as treatment modality, cisplatin was the main medication used [Haisfield-Wolfe et al., 2012; Xiao et al., 2013; Rosenthal et al., 2014; Eraj et al., 2017; Xiao et al., 2017; McDowell et al., 2018].
Considering the treatment-related toxicity assessment, 5 studies ( $45.4 \%$ ) assessed patients both during RT and after RT completion [Murphy et al., 2010; HaisfieldWolfe et al., 2012; Xiao et al., 2013; Kirca and Kutlutürkan, 2017; Barnhart et al., 2018], 3 studies (27.3\%) assessed patients after the conclusion of RT [Eraj et al., 2017; McDowell et al., 2018; Ridner et al., 2018], and 3 studies (27.3\%) assessed patients during the course of RT [Rosenthal et al., 2014; Xiao et al., 2017; Chiang et al., 2018]. For the classification of the observed toxicities, 5 studies (45.4\%) used the MD Anderson Symptom Inventory [Rosenthal et al., 2014; Eraj et al., 2017; Xiao et al., 2017; Chiang et al., 2018; McDowell et al., 2018], 2 studies (28.2\%) used The Memorial Symptom Assessment Scale [Haisfield-Wolfe et al., 2012; Kirca and Kutlutürkan, 2017], 1 (9.1\%) used the NCI Common Toxicity Criteria 2.0 [Xiao et al., 2013], 1 (9.1\%) used the Vanderbilt Head and Neck Symptom Survey [Murphy et al., 2010], 1 (9.1\%) used the Vanderbilt Head and Neck Symptom Survey version 2.0 [Ridner et al., 2018], and 1 (7.1\%) characterized the toxicities as present or absent [Barnhart et al., 2018].

Results of the risk of bias assessment are shown in Figure 2. Six studies (54.5\%) were classified as moderate risk of bias [Murphy et al., 2010; Haisfield-Wolfe et al., 2012; Rosenthal et al., 2014; Kirca and Kutlutürkan, 2017; Barnhart et al., 2018; Ridner et al., 2018], and 5 studies $(45.4 \%)$ were classified as low risk of bias [Xiao et al., 2013; Eraj et al., 2017; Xiao et al., 2017; Chiang et al., 2018; McDowell et al., 2018].

Since meta-analysis was not feasible due to the heterogeneity across studies, the quality of evidence was reported in a narrative summary of findings of GRADE and based on the study design of the included papers (online suppl. Table 5). The 9 descriptive studies provided weaker scientific evidence and had heterogeneous methodologies, resulting in a serious level of inconsistency. Also, moderate risk of bias in most studies downgraded it to a serious rate, leading to a low quality of evidence. The second outcome included only 2 studies and had fewer patients; however, they represented stronger level of evidence (cross sectional), had minor inconsistency across them, and had low risk of bias, leading to a moderate quality of evidence. Based on these results, further research may have an important impact on the estimate of these effects.

\section{Synthesis of Results}

From the selected studies, all $11(100 \%)$ reported symptoms such as difficulty in swallowing/dysphagia, dry 
Table 1. Main methodological data extracted from the studies included about the presence of radiation-related symptom clusters

\begin{tabular}{|c|c|c|c|c|}
\hline Study features & Study 1 & Study 2 & Study 3 & Study 4 \\
\hline Author & Murphy et al. & Haisfield-Wolfe et al. & Xiao et al. & Rosenthal et al. \\
\hline Country & US & US & US/Canada & US \\
\hline Journal & Head and Neck & Supportive Care in Cancer & Oral Oncology & Cancer \\
\hline Patients, $n$ & 235 & 21 & 684 & 149 \\
\hline Tumor topography & $\mathrm{HN}$ & oropharynx and larynx & $\begin{array}{l}\text { oral cavity, } \\
\text { oropharynx, } \\
\text { hypopharynx, or } \\
\text { larynx }\end{array}$ & $\begin{array}{l}\text { oral cavity, oropharynx, } \\
\text { nasopharynx, hypopharynx, } \\
\text { thyroid/trachea, major } \\
\text { salivary glands, nasal cavity/ } \\
\text { paranasal sinus, skin, } \\
\text { unknown }\end{array}$ \\
\hline Cancer treatment & CRT & RT or CRT & CRT & RT or CRT \\
\hline Mean radiation dose & $\mathrm{NI}$ & NI & $71.24 \mathrm{~Gy}$ & 66 Gy \\
\hline Type of RT & $\mathrm{NI}$ & $\mathrm{NI}$ & AFR or SFR & IMRT \\
\hline CT medication & NI & $\begin{array}{l}\text { cisplatin, taxol, Taxotere or } \\
\text { Erbitrux }\end{array}$ & cisplatin & $\begin{array}{l}\text { cisplatin, cetuximab, } \\
\text { multiagent regimens, and } \\
\text { carboplatin }\end{array}$ \\
\hline CT cycles & $\mathrm{NI}$ & NI & 2 or 3 & NI \\
\hline $\begin{array}{l}\text { Toxicities/time of } \\
\text { assessment }\end{array}$ & $\begin{array}{l}\text { patients receiving CRT up } \\
\text { to } 10-14 \text { months after } \\
\text { treatment }\end{array}$ & $\begin{array}{l}\text { 1st week of RT, middle of RT, end } \\
\text { of RT, } 6 \text { weeks post-RT }\end{array}$ & $\begin{array}{l}\text { end of } 1 \text { st CT, end of } \\
\text { 2nd CT, } 3 \text { months } \\
\text { after RT beginning }\end{array}$ & $\begin{array}{l}\text { pre-RT and weekly during the } \\
6-7 \text { week RT course }\end{array}$ \\
\hline Toxicity criteria & $\begin{array}{l}\text { Vanderbilt Head and } \\
\text { Neck Symptom Survey }\end{array}$ & $\begin{array}{l}\text { the memorial symptom assessment } \\
\text { scale }\end{array}$ & $\begin{array}{l}\text { NCI common toxicity } \\
\text { criteria (CTC) } 2.0\end{array}$ & $\begin{array}{l}\text { MD Anderson } \\
\text { Symptom Inventory-HN } \\
\text { module }\end{array}$ \\
\hline $\begin{array}{l}\text { Criteria for toxicity } \\
\text { inclusion }\end{array}$ & $\begin{array}{l}\text { results from a survey of } \\
\text { the most experienced } \\
\text { symptoms by } 26 \mathrm{HNC} \\
\text { patients submitted to CRT }\end{array}$ & $\begin{array}{l}11 \text { specific symptoms: } \\
\text { most problematic reported by the } \\
\text { patients }\end{array}$ & $\begin{array}{l}\text { symptoms with }>10 \% \\
\text { average prevalence } \\
\text { across the } 3 \text { time } \\
\text { points }\end{array}$ & $\begin{array}{l}\text { hierarchical cluster analysis of } \\
\text { symptoms at the end of } \\
\text { therapy }\end{array}$ \\
\hline
\end{tabular}


Table 1 (continued)

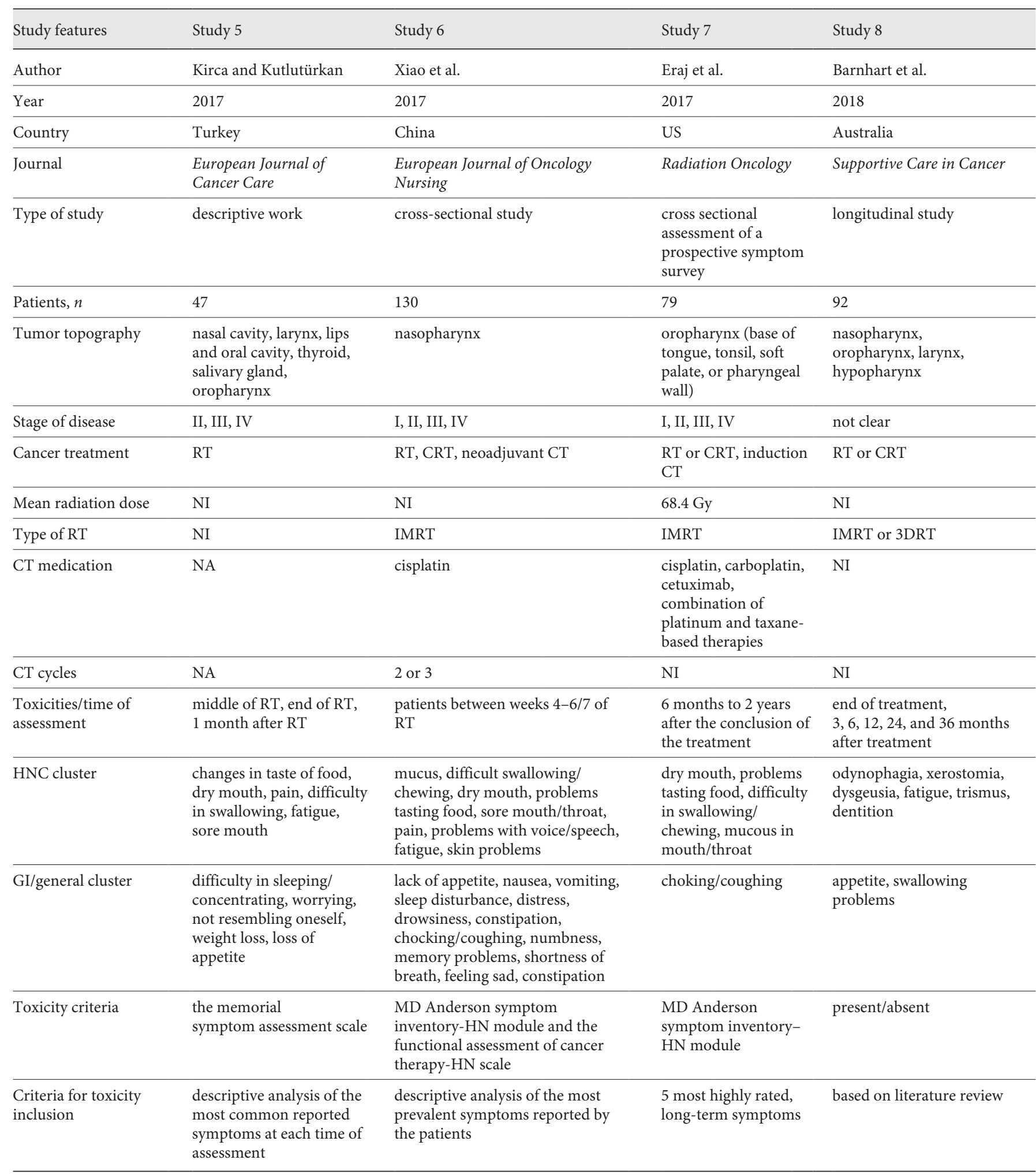


Table 1 (continued)

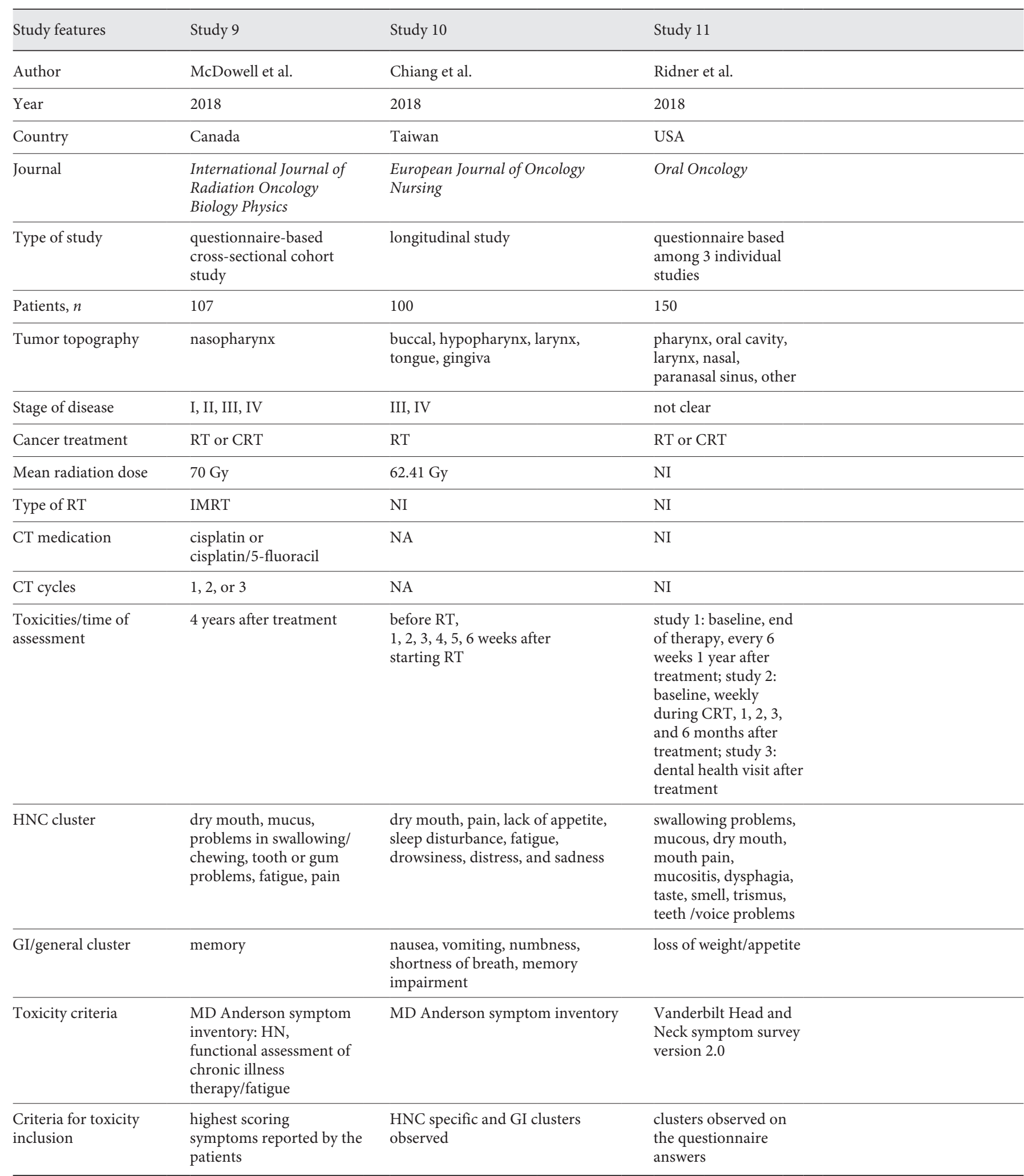

NA, not applied; NI, not informed; CRT, chemoradiotherapy; CT, chemotherapy; HN, head and neck; HNC, HN cancer; RT, radiotherapy; IMRT, intensity-modulated RT; AFR, accelerated fractionation RT; SFR, standard fractionation RT; 3DRT, 3D conformational RT. 


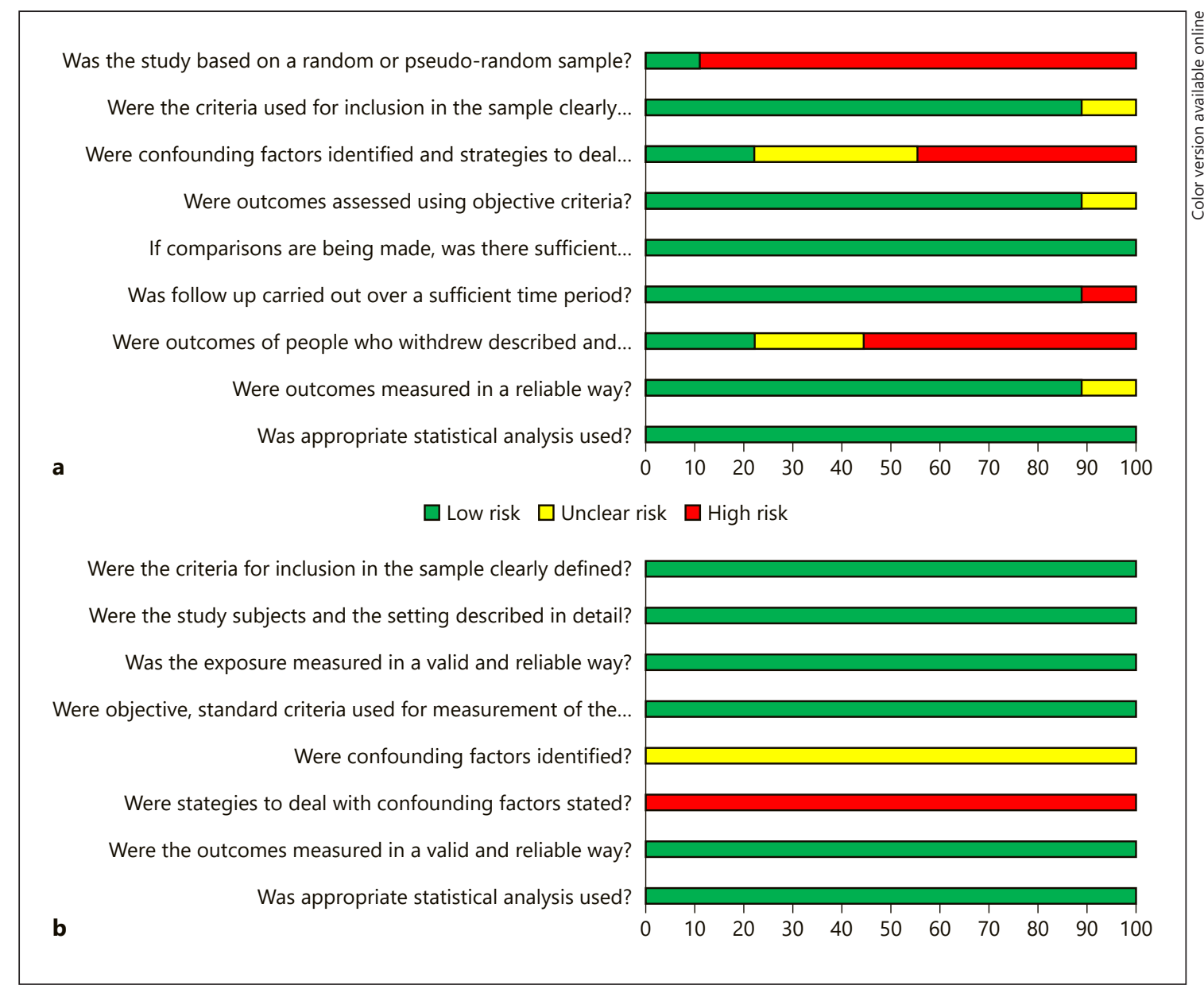

Fig. 2. Risk of bias in studies included about the symptom cluster among head-and-neck cancer patients. a MAStARI critical appraisal tools for descriptive/case series. b Joanna Briggs Institute critical appraisal checklist for analytical cross-sectional studies.

mouth/xerostomia, and pain [Murphy et al., 2010; Haisfield-Wolfe et al., 2012; Xiao et al., 2013; Rosenthal et al., 2014; Eraj et al., 2017; Kirca and Kutlutürkan, 2017; Xiao et al., 2017; Barnhart et al., 2018; Chiang et al., 2018; McDowell et al., 2018; Ridner et al., 2018], 8 studies (72.7\%) reported taste alterations [Murphy et al., 2010; Xiao et al., 2013; Rosenthal et al., 2014; Eraj et al., 2017; Kirca and Kutlutürkan, 2017; Xiao et al., 2017; Barnhart et al., 2018; Ridner et al., 2018], 7 studies (63.6\%) reported fatigue [Xiao et al., 2013; Rosenthal et al., 2014; Kirca and Kutlutürkan, 2017; Xiao et al., 2017; Barnhart et al., 2018; Chiang et al., 2018; McDowell et al., 2018], 5 studies (45.4\%) reported sore mouth [Murphy et al., 2010; Haisfield-Wolfe et al., 2012; Rosenthal et al., 2014; Kirca and Kutlutürkan, 2017; Xiao et al., 2017], 6 studies (54.5\%) reported problems with the presence of mucous on the mouth/throat [Murphy et al., 2010; Rosenthal et al., 2014; Eraj et al., 2017; Xiao et al., 2017; McDowell et al., 2018; Ridner et al., 2018], 4 studies (36.3\%) reported chewing problems [Rosenthal et al., 2014; Eraj et al., 2017; Xiao et al., 2017; McDowell et al., 2018], 3 studies (27.3\%) reported tooth/gum problems/dental caries [Barnhart et al., 2018; McDowell et al., 2018; Ridner et al., 2018], 3 studies $(27.3 \%)$ reported radiodermatitis [Haisfield-Wolfe et al., 2012; Xiao et al., 2013; Xiao et al., 2017], 2 studies (18.2\%) reported problems related to oral mucositis $(\mathrm{OM})$ [Xiao et al., 2013; Ridner et al., 2018], 2 studies (18.2\%) reported trismus [Barnhart et al., 2018; Ridner et al., 2018] and, finally, 1 study (9.1\%) reported smell alterations [Ridner et al., 2018]. Results of the distribution of HN-specific symptoms among the studies are shown in online supplementary Figure 1. 


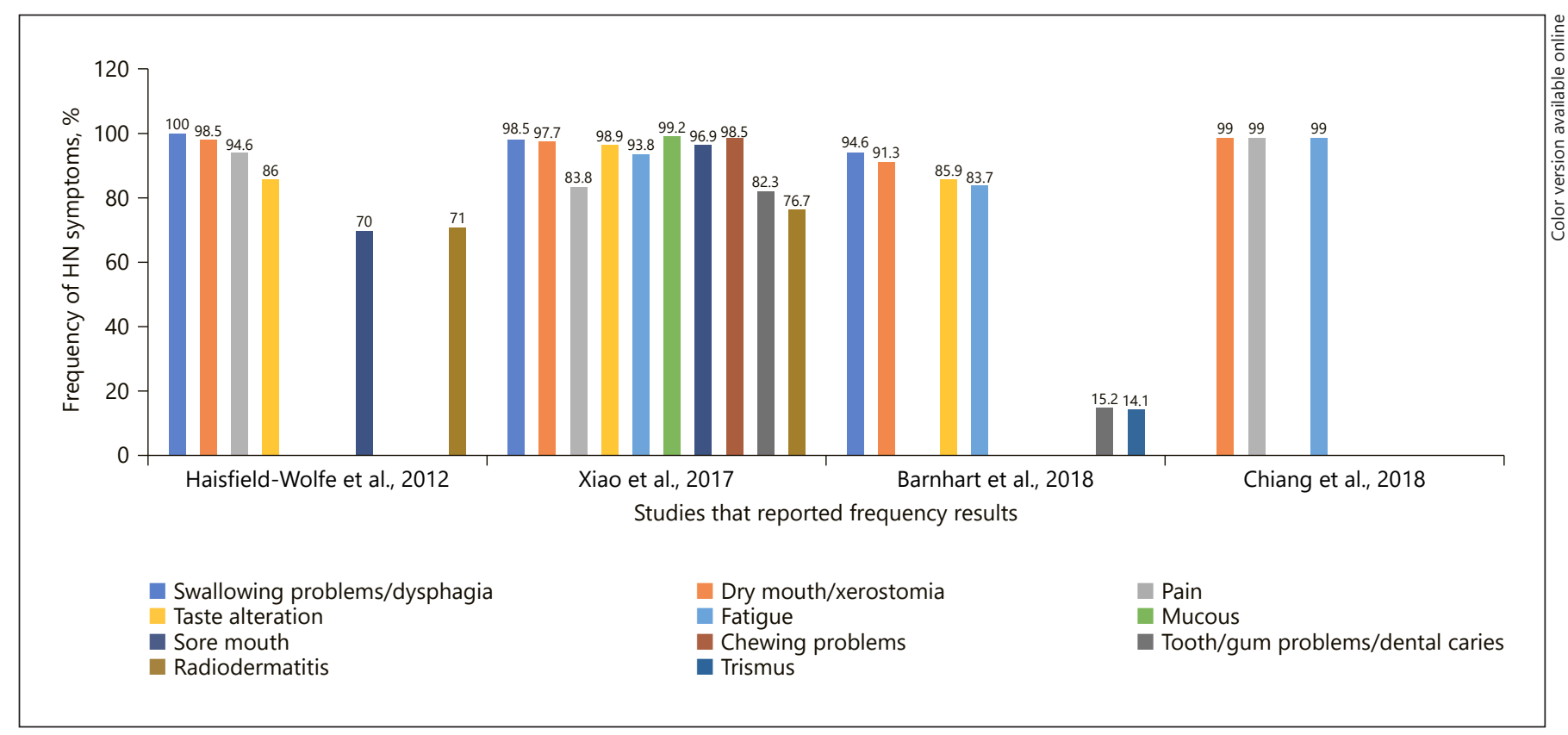

Fig. 3. Frequency of head-and-neck-specific $(\mathrm{HN})$ symptoms reported in the studies included.

Fig. 4. Frequency of gastrointestinal (GI) symptoms reported in the studies included.

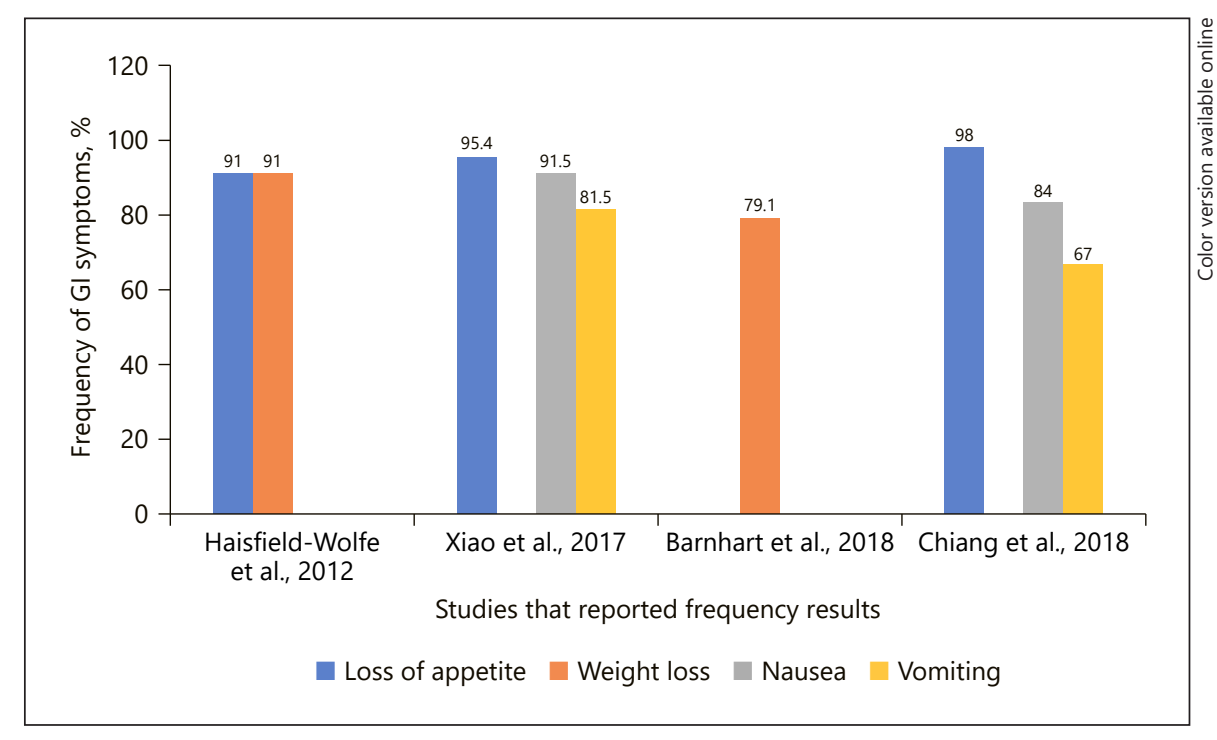

Results of the analysis of the presence of GI symptoms are shown in online supplementary Figure 2. Eight studies (72.7\%) reported loss of appetite [Murphy et al., 2010; Haisfield-Wolfe et al., 2012; Rosenthal et al., 2014; Kirca and Kutlutürkan, 2017; Xiao et al., 2017; Barnhart et al., 2018; Chiang et al., 2018; Ridner et al., 2018], 5 studies (45.4\%) reported weight loss [Murphy et al., 2010; Haisfield-Wolfe et al., 2012; Xiao et al., 2013; Kirca and Kutlutürkan, 2017; Ridner et al., 2018], 4 studies (36.3\%) re- ported nausea and vomiting [Xiao et al., 2013; Rosenthal et al., 2014; Xiao et al., 2017; Chiang et al., 2018], and 1 study (9.1\%) reported dehydration [Xiao et al., 2013].

The high heterogeneity in reporting the results observed in the studies included made it impossible to assess frequency and prevalence of treatment-related toxicities among HN cancer patients. Nevertheless, 4 studies (36.3\%) reported frequency values for $\mathrm{HN}$ and GI symptoms (Fig. 3, 4) [Haisfield-Wolfe et al., 2012; Xiao et al., 
2017; Barnhart et al., 2018; Chiang et al., 2018]. Swallowing problems/dysphagia were reported by 3 studies with a mean frequency of $97.7 \%$ for 243 patients [HaisfieldWolfe et al., 2012; Xiao et al., 2017; Barnhart et al., 2018]. Dry mouth/xerostomia was reported by all studies with a mean frequency of $94.75 \%$ for 343 patients [HaisfieldWolfe et al., 2012; Xiao et al., 2017; Barnhart et al., 2018; Chiang et al., 2018]. Pain was reported by 3 studies with a mean frequency of $91.3 \%$ for 151 patients [HaisfieldWolfe et al., 2012; Xiao et al., 2017; Chiang et al., 2018]. Taste alterations were reported by 3 studies with a mean frequency of $89.6 \%$ for 243 patients [Haisfield-Wolfe et al., 2012; Xiao et al., 2017; Barnhart et al., 2018]. Fatigue was reported by 3 studies with a mean frequency of $92.2 \%$ for 322 patients [Xiao et al., 2017; Barnhart et al., 2018; Chiang et al., 2018]. Mucous production was reported by 1 study with a frequency of $99.2 \%$ for 130 patients [Xiao et al., 2017]. Sore mouth was reported by 2 studies with a mean frequency of $83.5 \%$ for 151 patients [HaisfieldWolfe et al., 2012; Xiao et al., 2017]. Chewing problems were reported by 1 study with a frequency of $98.5 \%$ for 130 patients [Xiao et al., 2017]. Tooth/gum problems/ dental caries were reported by 2 studies with a mean frequency of $48.8 \%$ for 222 patients [Xiao et al., 2017; Barnhart et al., 2018]. Radiodermatitis was reported by 2 studies with a mean frequency of $73.9 \%$ for 151 patients [Haisfield-Wolfe et al., 2012; Xiao et al., 2017]. Trismus was reported by 1 study with a frequency of $14.1 \%$ for 92 patients [Barnhart et al., 2018]. Four studies reported lack of appetite with a mean frequency of $90.9 \%$ for 343 patients [Haisfield-Wolfe et al., 2012; Xiao et al., 2017; Barnhart et al., 2018; Chiang et al., 2018]. One study reported weight loss with a frequency of $91 \%$ for 21 patients [Haisfield-Wolfe et al., 2012]. Two studies reported nausea and vomiting with a mean frequency of 87.8 and $74.3 \%$, respectively, for 230 patients [Xiao et al., 2017; Chiang et al., 2018]. No studies reported frequency values for OM, smell alterations, and dehydration. Detailed information on the results reported from the studies included are available in online supplementary Table 6.

\section{Symptom Clusters in Patients with HNC}

Results from the present systematic review described several clusters of symptoms following HNC treatment, which include specific $\mathrm{HN}$ conditions, such as dry mouth, dysphagia, pain, taste disturbances, fatigue, $\mathrm{OM}$, and radiodermatitis, and GI manifestations, such as nausea, vomiting, and dehydration [Murphy et al., 2010; Haisfield-Wolfe et al., 2012; Xiao et al., 2013; Rosenthal et al., 2014; Eraj et al., 2017; Kirca and Kutlutürkan, 2017; Xiao et al., 2017; Barnhart et al., 2018; Chiang et al., 2018; McDowell et al., 2018; Ridner et al., 2018]. This clustering of oral symptoms using contemporary concepts brought new ideas for the analysis of RRC pathogenesis and the impact of dietary changes, deficient oral hygiene, and the highly cariogenic oral environment on the dentition of HNC survivors (Fig. 5).

\section{Discussion}

HNRT is known to cause several acute and chronic toxicities in the oral cavity. Within the first 3 weeks, patients undergoing HNRT experience a series of symptoms that burden, evolve, and overlap. They often develop OM, radiation dermatitis, edema, dysgeusia, and a shift in the oral microbiota composition [Murphy et al., 2010; Xiao et al., 2013; Chiang et al., 2018; Ridner et al., 2018]. Additionally, these patients may develop associated pain, copious mucous production, hyposalivation, xerostomia, and acute tissue swelling, which contribute to acute dysphagia [Murphy et al., 2010; Haisfield-Wolfe et al., 2012; Xiao et al., 2013; Eraj et al., 2017; Xiao et al., 2017; Barnhart et al., 2018; Chiang et al., 2018; McDowell et al., 2018; Ridner et al., 2018]. Late effects include skin and salivary gland fibrosis, lymphedema, and damage to neural structures, hyposalivation, trismus, dysphagia, RRC, and osteoradionecrosis [Kielbassa et al., 2006; Eraj et al., 2017; Barnhart et al., 2018; McDowell et al., 2018]. Adverse effects of cancer treatment represent profound and long-lasting functional alterations and diminished quality of life, which comprise a complex network of interrelated factors that include functional, biological, psychological, and social components [Murphy et al., 2007; Murphy and Gilbert, 2009; Ridner et al., 2018].

The symptoms experienced by HNC patients are broad in scope and encompass both local and systemic symptoms. Furthermore, instead of occurring in isolation, results observed in the present systematic review indicate that they occur in clusters, exacerbating the overall symptom experience. "Symptom clusters" are defined as groups of at least 2 or 3 concurrent symptoms that are synergistically interrelated [Murphy et al., 2007; Xiao et al., 2013; Dong et al., 2014]. Two main distinct and stable clusters were described for HNC patients, identified through factor modeling among 10 identified treatment-related symptoms: $\mathrm{HN}$-specific symptom clusters (encompassing mucositis; radiodermatitis; pain; dysphagia; taste disturbances; dry mouth, and fatigue) and GI clusters (nausea, vomiting, and dehydration) [Aguiar et al., 2009; Silva et 


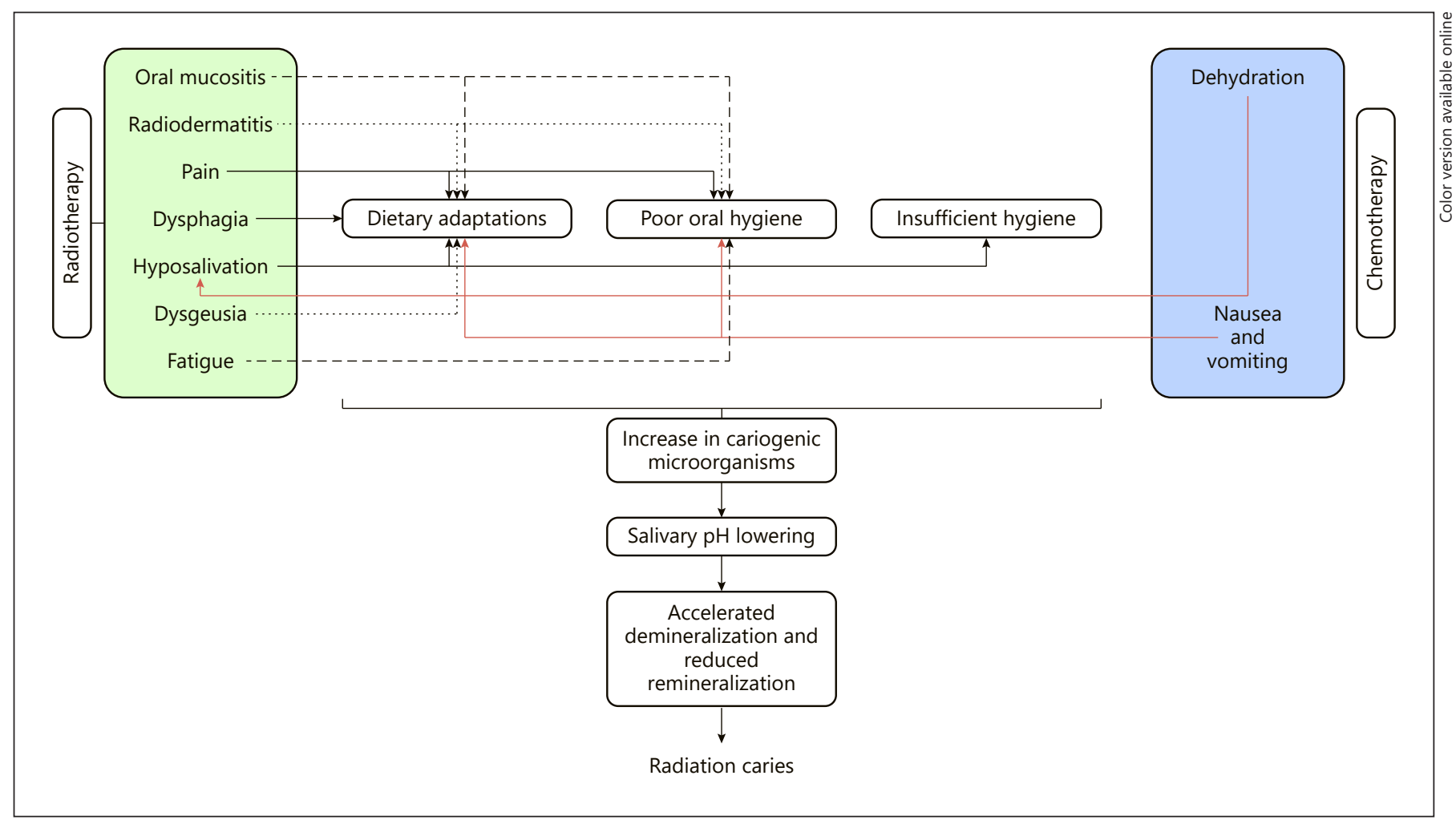

Fig. 5. Flowchart presenting the interactions between the head and neck (green) and the gastrointestinal symptom clusters (blue) in RRC pathogenesis.

al., 2009; Murphy et al., 2010; Haisfield-Wolfe et al., 2012; Xiao et al., 2013; Eraj et al., 2017; Xiao et al., 2017; Barnhart et al., 2018; Chiang et al., 2018; McDowell et al., 2018; Ridner et al., 2018]. These symptom clusters may be associated with the development of a highly cariogenic oral environment and the lack of proper oral hygiene leading to the onset and development of RRC [Cohen et al., 2016].

Dysphagia is defined as difficulty in swallowing and can be an acute or late result of HNRT. Acute dysphagia is associated with mucosa and soft tissue damage within the treatment field particularly because of OM, radiation dermatitis, and edema of the soft tissue. Pain, hyposalivation associated with thickened and more viscous mucous production, and tissue swelling contribute to acute dysphagia. Late dysphagia is the result of tissue fibrosis and stiffness due to the ongoing inflammatory cytokine cascade effects, as well as to lymphedema and radiation-induced damage to neural structures. Patients suffer from aspiration and choking, and may consciously or unconsciously alter the type and consistency of food that they eat, resulting in nutritional deficiencies and an oral environment favorable for RRC onset and progression [Mur- phy et al., 2007; Nevens et al., 2017; Ridner et al., 2018; Santa Cruz et al., 2018].

In HNC patients, dry mouth (xerostomia) is caused by hyposalivation due to radiogenic effects on salivary glands. It has a rapid onset, and it is the most common persistent oral side effect for patients receiving HNRT [Sciubba and Goldenberg, 2006]. Saliva becomes scant and thicker causing difficulties in speaking and inducing taste alterations as well as distress in chewing and swallowing. This scenario has an influence on dietary alterations, leading to the intake of softer and more carbohydrate-rich food [Aguiar et al., 2009]. Besides the quantitative effects, qualitative salivary changes also occur unleashing an imbalance in its ionic composition. Consequently, its buffering and tooth remineralization capacity are reduced, leading to loss of the demineralization/remineralization equilibrium and facilitating the more rapid loss of minerals from dentin and enamel following RT [Murphy and Gilbert, 2000; Marsh, 2003; Barnhart et al., 2018; Ridner et al., 2018].

In addition, an imbalance in both salivary organic components (glycoproteins and proteins) and in adaptive 
and innate immunity occurs following HNRT, altering the establishment and selection of the oral microbiota present on oral hard and soft tissues. Also, the frequent sugar and carbohydrate-rich food intake creates regular conditions of low $\mathrm{pH}$ within the dental biofilm and selects for acidogenic and aciduric bacteria such as mutans streptococci and lactobacilli, predisposing the enamel - which is known for being highly porous and permeable after HNC treatment [Madrid et al., 2017] - to the rapid onset and progression of RRC. In other words, a real "ecological catastrophe" occurs in the oral cavity of cancer patients following HNRT due to the disruption of the natural balance that normally exists in the mouth between the microbiota and the host, and which drives dysbiotic changes in the composition of the biofilm, thereby creating a favorable environment for RRC [Marsh, 2003].

Pain is a ubiquitous problem faced by all HNC patients due to the tumor before therapy begins, and up to $76 \%$ of patients suffer from severe pain related to acute therapy toxicities such as $\mathrm{OM}$ and radiodermatitis despite the use of opioids [Murphy et al., 2007]. After treatment completion, they experience pain when doing several basic physical functions due to fibrosis, muscle loss, neck dissection, and neural impairment. Pain significantly affects function, with high percentages of patients reporting difficulties in swallowing, eating, drinking, talking, sleeping, and maintaining daily self-care functions such as oral hygiene [Murphy and Gilbert, 2000; Xiao et al., 2017; Chiang et al., 2018; Ridner et al., 2018].

All HNC patients undergoing cancer therapy experience taste disturbances. It is caused by a multitude of other toxicities including $\mathrm{OM}$, deficient oral hygiene, a shift in their oral microbiota, taste buds, and oral neural structure impairment, medication or chemotherapies, and especially salivary flow decrease [Sciubba and Goldenberg, 2006; Murphy et al. 2007; Barnhart et al., 2018; Ridner et al., 2018]. It markedly impairs a patient's quality of life, leading to decreased food intake and a switch to sweeter foods (the most maintained flavor reported by the patients). Unfortunately, intake of carbohydrate-rich and sweeter foods provides a highly cariogenic environment and fosters RRC development and rapid progression [Aguiar et al., 2009].

Fatigue is another well-documented side effect observed in patients undergoing RT. The lack of appetite, mainly due to the presence of chemosensory dysfunction, such as taste and smell dysfunction, can result in patients' general deconditioning which may lead to profound weight loss, with a decrease in lean and fat body mass, and individuals experiencing weakness and fatigue [Murphy et al., 2010; Haisfield-Wolfe et al., 2012; Xiao et al., 2013;
Kirca and Kutlutürkan, 2017; Ridner et al., 2018]. This occurs due to chemotherapy and radiation-induced metabolic changes; impaired food intake caused by pain, tumor-related factors, dysphagia, socioeconomic difficulties impairing the purchase of nutritional supplements, and even depression [Murphy et al., 2007; Murphy and Gilbert, 2009]. All of these events compound a complex network leading to a decrease in physical functioning and loss of the ability to conduct daily activities such as proper oral hygiene, further enhancing RRC.

HNC patients that undergo RT will develop OM, especially when RT is associated with concurrent chemotherapy. The site of OM development depends on the tumor site, tumor size, and treatment planning, but in any case it produces mucosal pain and swelling, leading to bleeding, difficulty in speaking, sleeping, or mouth opening; dysphagia, and anorexia. In addition, it leads to dietary adaptations with a switch to softer and carbohydrate-rich foods at an increased intake. This fact, associated with an impaired or absent oral hygiene, produces an environment conducive to RRC onset [Murphy and Gilbert, 2000; Aguiar et al., 2009; Xiao et al., 2013; Ridner et al., 2018].

Radiodermatitis causes wounds, pain, and a burning sensation on the skin included in the treatment field. The radiogenic soft tissue damage may also affect the local lymphatic structures and muscles, being associated in the long term with lymphedema, cutaneous and muscular fibrosis, and consequent trismus. In this way, besides the swallowing difficulties, patients present distress on opening the mouth and must change their dietary habits to softer and more cariogenic food, which combined with the additional impairment in proper oral hygiene due to pain and trismus increases their risk of RRC [Murphy and Gilbert, 2009; Nevens et al., 2017; Ridner et al., 2018; Santa Cruz et al., 2018].

Systemic symptom clusters associated with HNC treatment toxicities were described by Xiao et al. [2013] as a stable identified GI cluster involving nausea, vomiting, and dehydration often induced by CT or CRT. We go further and suggest that this "GI cluster" may have a significant impact on RCC pathophysiology, especially due to recurrent vomiting, which may result in dehydration and intensifies hyposalivation, lowering the protective salivary effects against caries. In addition, vomiting may produce a lower oral $\mathrm{pH}$, leading to an elevated risk of enamel and dentin dissolution. All of the side effects associated with nausea create an additional obstacle for proper oral hygiene in $\mathrm{HNC}$ patients and represent a favorable environment for the onset and development of RRC. 
Fig. 6. Oral health status in 2 head-and-neck cancer patients examined before radiotherapy resembling radiation-related caries patients. a Note the poor oral hygiene, extensive carious lesions, brown-blackish color pigmentation due to smoking habits, and extensive tooth loss. b Presence of extensive periodontal disease, tooth loss, severe caries, and multiple residual roots - one of them (in the lower right mandibular area) presenting signs of apical periodontitis.
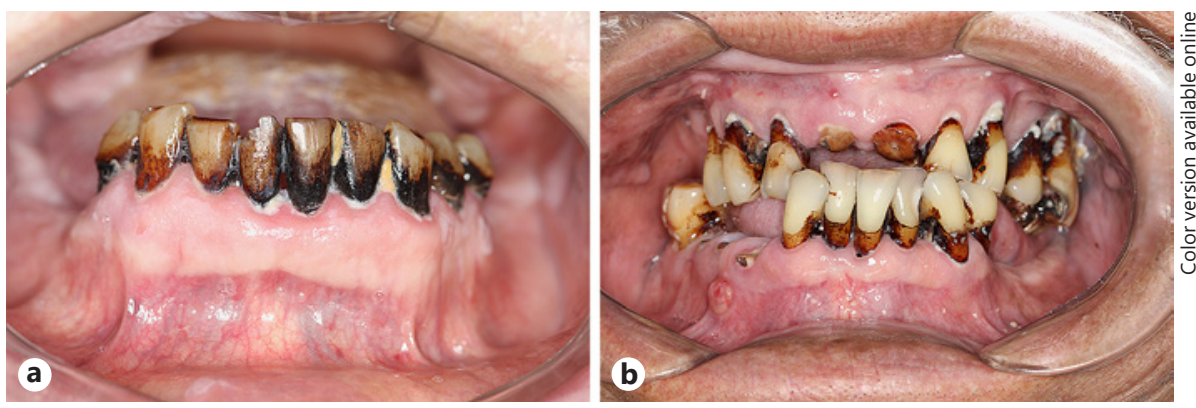

Lastly, it is relevant to mention that most of the oral cancer patients are poorly educated, low-income individuals, with minimal oral hygiene and level of dental awareness. Many of these patients had never undergone dental treatment, and previous studies have demonstrated that nearly all the HNC patients examined just before HNRT need extensive dental care due to advanced periodontal disease, residual roots, and caries (Fig. 6) [Jham et al., 2008]. These complex psychosocial and behavioral features of HNC patients create a poor oral health scenario even before HNRT [Jham et al., 2008], which might be considered another pillar to the development and rapid progression of RRC.

\section{Strengths and Limitations}

Main strengths of this systematic review were rigorous searching and assessment methods and homogeneity in study objectives. Nonetheless, we found limitations such as heterogeneity of studies that met inclusion criteria regarding the methodology and criteria for toxicity assessment and report of observed results. In addition, frequency values were possible to be obtained for only 4 from the 11 studies included and, unfortunately, none of the papers included correlated the presence of symptoms or symptom clusters with the onset and progression of RRC, which made it impossible to perform a meta-analysis of the correlation of oral symptom clusters and RRC.

\section{Conclusions}

This review is the first to explore symptom clusters in HNC patients and their possible impact on RRC development and progression. HNC patients seldom present with a single oral symptom; thus, the understanding and managing of the specific conditions of the HN and GI symptom cluster manifestations may be of paramount importance for the preservation of cancer survivors' quality of life. Remarkably, there is evidence that the observed HN and GI symp- tom clusters may indirectly contribute to RRC onset and progression. This scenario composes a much more complex panorama than what has previously been suggested in terms of RRC pathogenesis and should be considered pivotal for RRC progression. Therefore, contemporary protocols of RRC prevention and treatment must take this broader HNRT-associated clustering of toxicities into account.

\section{Statement of Ethics}

The authors have no ethical conflicts to disclose.

\section{Disclosure Statement}

The authors declare that there are no conflicts of interest.

\section{Funding Sources}

The authors would like to gratefully acknowledge the financial support of the Coordination for the Improvement of Higher Education Personnel through the National Post Doctoral Program (CAPES/PNPD, Brazil), No. 1724203; São Paulo Research Foundation (FAPESP, Brazil), Nos. 2013/18402-8, 2016/22059-5, 2018/02233-6, and 2018/04657-8; and the National Council for Scientific and Technological Development (CNPq, Brazil).

\section{Author Contributions}

Adriele Ferreira Gouvêa Vasconcellos, Natália Rangel Palmier and Alan Roger Santos-Silva performed the systematic review methodology process and wrote the manuscript in consultation with Adriana Franco Paes Leme and Philip D. Marsh. Ana Gabriela Costa Normando and Mario Fernando de Goes performed risk of bias analysis within and across studies (GRADE). Thaís Bianca Brandão, Marcio Ajudarte Lopes, and Ana Carolina Prado Ribeiro designed this study. Karina Morais-Faria, Wagner Gomes-Silva, and Aljomar José Vechiato Filho drafted the manuscript and designed the figures. All authors discussed the results and commented on the manuscript. 


\section{References}

Aguiar GP, Jham BC, Magalhães CS, Sensi LG, Freire AR. A review of the biological and clinical aspects of radiation caries. J Contemp Dent Pract. 2009 Jul;10(4):83-9.

Barnhart MK, Robinson RA, Simms VA, Ward EC, Cartmill B, Chandler SJ, et al. Treatment toxicities and their impact on oral intake following non-surgical management for head and neck cancer: a 3-year longitudinal study. Support Care Cancer. 2018 Jul;26(7):234151.

Chiang SH, Ho KY, Wang SY, Lin CC. Change in symptom clusters in head and neck cancer patients undergoing postoperative radiotherapy: A longitudinal study. Eur J Oncol Nurs. 2018 Aug;35:62-6.

Cohen EE, LaMonte SJ, Erb NL, Beckman KL, Sadeghi N, Hutcheson KA, et al. American Cancer Society Head and Neck Cancer Survivorship Care Guideline. CA Cancer J Clin. 2016 May;66(3):203-39.

Dong ST, Butow PN, Costa DS, Lovell MR, Agar M. Symptom clusters in patients with advanced cancer: a systematic review of observational studies. J Pain Symptom Manage. 2014 Sep;48(3):411-50.

Eraj SA, Jomaa MK, Rock CD, Mohamed AS, Smith BD, Smith JB, et al.; MD Anderson Head and Neck Cancer Symptom Working Group. Long-term patient reported outcomes following radiation therapy for oropharyngeal cancer: cross-sectional assessment of a prospective symptom survey in patients $\geq 65$ years old. Radiat Oncol. 2017 Sep;12(1):150.

Faria KM, Brandão TB, Ribeiro AC, Vasconcellos AF, de Carvalho IT, de Arruda FF, et al. Micromorphology of the dental pulp is highly preserved in cancer patients who underwent head and neck radiotherapy. J Endod. 2014 Oct;40(10):1553-9.

Gomes-Silva W, Prado Ribeiro AC, de Castro Junior G, Salvajoli JV, Rangel Palmier N, Lopes MA, et al. Head and neck radiotherapy does not increase gelatinase (metalloproteinase-2 and -9) expression or activity in teeth irradiated in vivo. Oral Surg Oral Med Oral Pathol Oral Radiol. 2017a Aug;124(2):175-82.

Gomes-Silva W, Prado-Ribeiro AC, Brandão TB, Morais-Faria K, de Castro Junior G, Mak MP, et al. Postradiation Matrix Metalloproteinase-20 Expression and Its Impact on Dental Micromorphology and Radiation-Related Caries. Caries Res. 2017b;51(3):216-24.

Haisfield-Wolfe ME, McGuire DB, Soeken K, Geiger-Brown J, De Forge B, Suntharalingam M. Prevalence and correlates of symptoms and uncertainty in illness among head and neck cancer patients receiving definitive radiation with or without chemotherapy. Support Care Cancer. 2012 Aug;20(8):1885-93.

Jham BC, Reis PM, Miranda EL, Lopes RC, Carvalho AL, Scheper MA, et al. Oral health status of 207 head and neck cancer patients before, during and after radiotherapy. Clin Oral Investig. 2008 Mar;12(1):19-24.
Kielbassa AM, Hinkelbein W, Hellwig E, MeyerLückel H. Radiation-related damage to dentition. Lancet Oncol. 2006 Apr;7(4):326-35.

Kırca K, Kutlutürkan S. Symptoms of patients with head and neck cancers undergoing radiotherapy. Eur J Cancer Care (Engl). 2017 Nov;26(6):e12584.

Lieshout HF, Bots CP. The effect of radiotherapy on dental hard tissue-a systematic review. Clin Oral Investig. 2014 Jan;18(1):17-24.

Madrid CC, de Pauli Paglioni M, Line SR, Vasconcelos KG, Brandão TB, Lopes MA, et al. Structural Analysis of Enamel in Teeth from Head-and-Neck Cancer Patients Who Underwent Radiotherapy. Caries Res. 2017; 51(2):119-28

Marsh PD. Are dental diseases examples of ecological catastrophes? Microbiology. 2003 Feb; 149(Pt 2):279-94.

McDowell LJ, Rock K, Xu W, Chan B, Waldron J, Lu L, et al. Long-Term Late Toxicity, Quality of Life, and Emotional Distress in Patients With Nasopharyngeal Carcinoma Treated With Intensity Modulated Radiation Therapy. Int J Radiat Oncol Biol Phys. 2018 Oct; 102(2):340-352.

Moher D, Liberati A, Tetzlaff J, Altman DG; PRISMA Group. Preferred reporting items for systematic reviews and meta-analyses: the PRISMA statement. PLoS Med. 2009 Jul; 6(7):e1000097.

Murphy BA, Dietrich MS, Wells N, Dwyer K, Ridner SH, Silver HJ, et al. Reliability and validity of the Vanderbilt Head and Neck Symptom Survey: a tool to assess symptom burden in patients treated with chemoradiation. Head Neck. 2010 Jan;32(1):26-37.

Murphy BA, Gilbert J, Ridner SH. Systemic and global toxicities of head and neck treatment. Expert Rev Anticancer Ther. 2007 Jul;7(7): 1043-53.

Murphy BA, Gilbert J. Dysphagia in head and neck cancer patients treated with radiation: assessment, sequelae, and rehabilitation. Semin Radiat Oncol. 2009 Jan;19(1):35-42.

Murphy BA, Gilbert J. Oral cancers: supportive care issues. Periodontol 2000. 2011 Oct;57(1): $118-31$.

Nevens D, Duprez F, Daisne JF, Laenen A, De Neve W, Nuyts S. Radiotherapy induced dermatitis is a strong predictor for late fibrosis in head and neck cancer. The development of a predictive model for late fibrosis. Radiother Oncol. 2017 Feb;122(2):212-6.

Palmier NR, Gouvea AF, Santos-Silva AR. The impact of clustering of oral symptoms in the pathogenesis of radiation caries: a systematic review. PROSPERO 2019 CRD42019132709. Available from: https://www.crd.york.ac.uk/ prospero/display_record.php?ID=CRD 42019132709.

Ribeiro AC, Lopes MA, Brandão TB, Santos-Silva AR. Clustering of oral symptoms versus radiation-induced apical periodontitis. Clin Oral Investig. 2013 Jan;17(1):337.
Ridner SH, Rhoten BA, Niermann KJ, Murphy BA, Dietrich MS. Vanderbilt head and neck symptom survey, version 2.0: Clinical and research utility for identification of symptom clusters and changes in symptoms over time. Oral Oncol. 2018 Aug;83:25-31.

Rosenthal DI, Mendoza TR, Fuller CD, Hutcheson KA, Wang XS, Hanna EY, et al. Patterns of symptom burden during radiotherapy or concurrent chemoradiotherapy for head and neck cancer: a prospective analysis using the University of Texas MD Anderson Cancer Center Symptom Inventory-Head and Neck Module. Cancer. 2014 Jul;120(13):1975-84.

Santa Cruz O, Tsoutsou P, Castella C, Khanfir K, Anchisi S, Bouayed S, et al. Locoregional Control and Toxicity in Head and Neck Carcinoma Patients following Helical TomotherapyDelivered Intensity-Modulated Radiation Therapy Compared with 3D-CRT Data. Oncology. 2018;95(2):61-8.

Sciubba JJ, Goldenberg D. Oral complications of radiotherapy. Lancet Oncol. 2006 Feb;7(2): $175-83$.

Santos-Silva AR, Feio PS, Vargas PA, Correa ME, Lopes MA. cGVHD-related caries and its shared features with others 'dry-mouth'- related caries. Braz Dent J. 2015 Jul-Aug;26(4):435-40.

Silva AR, Alves FA, Antunes A, Goes MF, Lopes MA. Patterns of demineralization and dentin reactions in radiation-related caries. Caries Res. 2009;43(1):43-9.

Springer IN, Niehoff P, Warnke PH, Böcek G, Kovács G, Suhr M, et al. Radiation caries-radiogenic destruction of dental collagen. Oral Oncol. 2005 Aug;41(7):723-8.

Sroussi HY, Epstein JB, Bensadoun RJ, Saunders DP, Lalla RV, Migliorati CA, et al. Common oral complications of head and neck cancer radiation therapy: mucositis, infections, saliva change, fibrosis, sensory dysfunctions, dental caries, periodontal disease, and osteoradionecrosis. Cancer Med. 2017 Dec;6(12):2918-31.

The Joanna Briggs Institute. The Joanna Briggs Institute Reviewer's Manual 2014 Edition: Meta Analysis of Statistics Assessment and Review Instrument (MAStARI) critical appraisal tools Comparable cohort/ Case control studies. Adelaide, Australia: The Joanna Briggs Institute; 2014.

Xiao C, Hanlon A, Zhang Q, Ang K, Rosenthal DI, Nguyen-Tan PF, et al. Symptom clusters in patients with head and neck cancer receiving concurrent chemoradiotherapy. Oral Oncol. 2013 Apr;49(4):360-6.

Xiao C, Hanlon A, Zhang Q, Movsas B, Ang K, Rosenthal DI, et al. Risk factors for clinicianreported symptom clusters in patients with advanced head and neck cancer in a phase 3 randomized clinical trial: RTOG 0129. Cancer. 2014 Mar; 120(6):848-54.

Xiao W, Chan CW, Fan Y, Leung DY, Xia W, He $\mathrm{Y}$, et al. Symptom clusters in patients with nasopharyngeal carcinoma during radiotherapy. Eur J Oncol Nurs. 2017 Jun;28:7-13. 